\title{
TRANSFORMER BASED DELIBERATION FOR TWO-PASS SPEECH RECOGNITION
}

\author{
Ke Hu, Ruoming Pang, Tara N. Sainath, Trevor Strohman \\ Google, Inc., USA \\ $\{$ huk, rpang, tsainath, strohman\}@google.com
}

\begin{abstract}
Interactive speech recognition systems must generate words quickly while also producing accurate results. Two-pass models excel at these requirements by employing a first-pass decoder that quickly emits words, and a second-pass decoder that requires more context but is more accurate. Previous work has established that a deliberation network can be an effective second-pass model. The model attends to two kinds of inputs at once: encoded audio frames and the hypothesis text from the first-pass model. In this work, we explore using transformer layers instead of long-short term memory (LSTM) layers for deliberation rescoring. In transformer layers, we generalize the "encoder-decoder" attention to attend to both encoded audio and first-pass text hypotheses. The output context vectors are then combined by a merger layer. Compared to LSTM-based deliberation, our best transformer deliberation achieves $7 \%$ relative word error rate improvements along with a $38 \%$ reduction in computation. We also compare against non-deliberation transformer rescoring, and find a $9 \%$ relative improvement.
\end{abstract}

Index Terms- Transformer, deliberation network, rescoring, two-pass automatic speech recognition

\section{INTRODUCTION}

End-to-end (E2E) automatic speech recognition (ASR) has made rapid progress in recent years $[1,2,3,4,5,6,7]$. Representative models include streaming models such as the recurrent neural network transducer (RNN-T) [1], attention-based models [8, 2, 3], and transformer-based models [9, 10, 11, 12]. Compared to sophisticated conventional models [13, 14], E2E models such as RNN-T and Listen, Attend and Spell (LAS) have shown competitive performance [6, 5, 7, 15]. To further improve recognition accuracy, a two-pass LAS rescoring model has been proposed in [16], which uses a nonstreaming LAS decoder to rescore the RNN-T hypotheses. The rescorer attends to audio encoding from the encoder to re-rank the first-pass hypotheses. [7] shows that by using an RNN-T model which is capable of endpointing based on an end-of-query token, LAS rescoring can outperform a large conventional model [13] in both latency and word error rate (WER).
Recently, deliberation network [17] has been proposed for second-pass rescoring [18] and achieved state-of-the-art WER on Google Voice Search (VS). By using multi-source attention in a LAS decoder, deliberation attends to both encoder outputs and first-pass hypotheses for rescoring. For comparison, LAS rescoring only attends to encoder outputs for rescoring [16, 7], and neural correction models post-process hypotheses using only text hypotheses or lattice $[19,20,21]$.

While long short-term memory (LSTM) has been a popular building block for E2E models, there has been a continuing success in applying transformer models [22] in ASR [23, 11, 10, 9, 24, 25, 4]. Instead of using a recurrent mechanism to model temporal dynamics, the transformer uses multi-headed attention to associate sequential elements in one step. $[23,11]$ incorporate transformer layers to conventional models for acoustic modeling. For E2E models, the transformer has been adapted or applied to streaming models $[10,9,12]$ and non-streaming models [4]. Comparative studies [26, 4] show that transformer-based models outperform their recurrent neural network (RNN) counterparts in a number of tasks. Transformers have also been applied in post-processing for E2E models. [25, 15] use transformer for spelling correction. [24] applies transformer decoder in second-pass rescoring. The non-autoregressive nature of a transformer layer enables token-level parallel rescoring, i.e., the rescoring of one token in a sequence does not depend on the previous one. This can significantly reduce latency on a matrix computation friendly hardware such as Tensor Processing Units (TPU).

In this work, we develop a transformer-based deliberation rescorer, which is more TPU friendly than its LSTM counterpart [18]. We use a transformer decoder in the second pass rescoring, i.e., the first-pass RNN-T model generates first-pass hypotheses, and then we use a transformer decoder to rescore the first-pass hypotheses. In rescoring, the transformer "encoder-decoder" attention attends to two sources: Encoder outputs and first-pass hypothesis encoding. The resultant context vectors are then combined by a merger layer to produce a new context vector that has the same dimension as the output from the previous self-attention layer. Our model is different from transformer rescoring [24] where the decoder only attends to the encoder outputs. As in [18], our model en- 


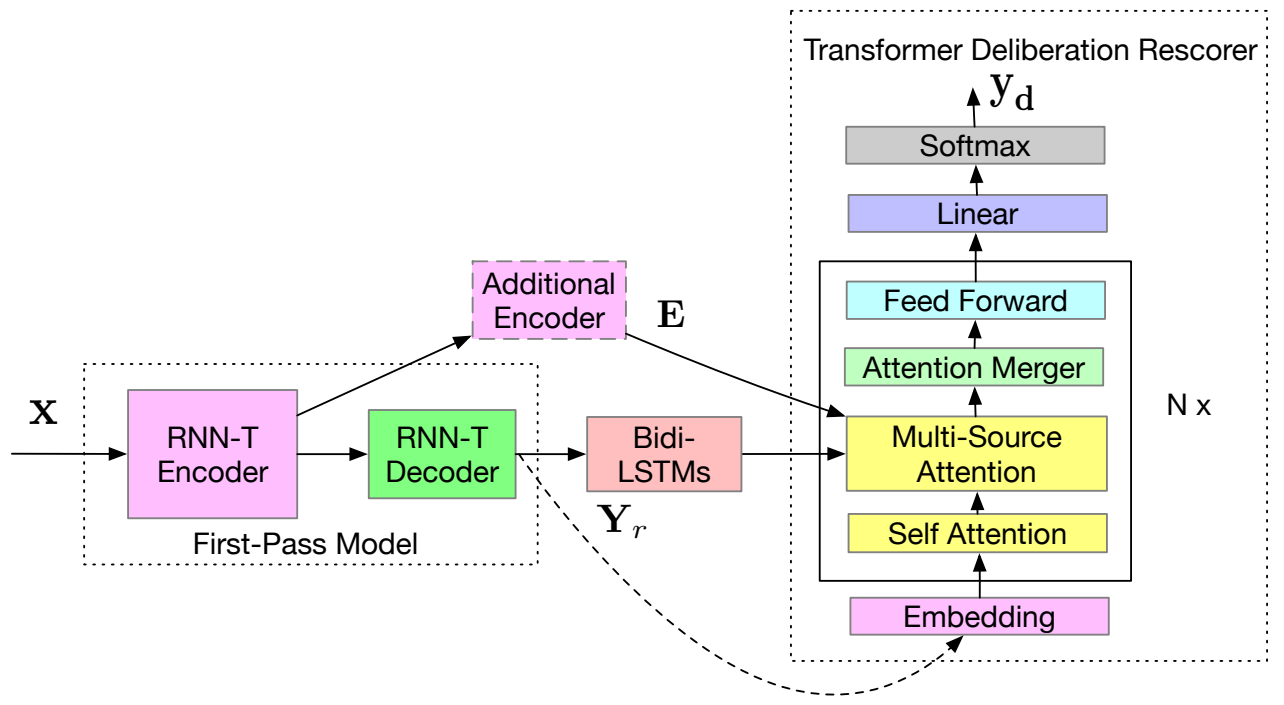

Fig. 1. Diagram of a two-pass transformer deliberation model with an optional additional encoder (dashed box). The RNN-T model generates first-pass hypotheses, and a second-pass transformer deliberation rescorer re-ranks the first-pass results. The "encoder-decoder" attention of the transformer attends to two sources: Acoustic encoding and first-pass hypothesis encoding. The two output context vectors are combined using a merger layer. In addition, first-pass hypotheses are bidirectionally encoded to extract context information.

codes first-pass hypotheses bidirectionally to model context. When encoding multiple hypotheses, we model the hypothesis order (i.e., rank in the n-best list) using a learned embedding [27] and add that to the embedding of wordpiece tokens in the corresponding hypothesis. We note that the wordpiecebased [28] sequences are usually short for VS queries, and computation can be paralleled to improve latency.

We conduct experiments on the same training data as in $[29,18,30]$, which is from multiple domains including Voice Search, YouTube, Telephony such as SwitchBoard [31] and Fisher [32], etc. We researched different types of merge layer, i.e., sum, concatenation, attention or gated averaging, to combine the attention vectors from encoder outputs and firstpass hypothesis encoding, and find sum is a more effective and efficient method. Similar to the previous study [18], we observe that using multiple RNN-T hypotheses, additional encoder (AE) layers, and minimum WER (MWER) training [33] improves WER. Order embedding and joint training further improve WER. In summary, our best proposed transformer deliberation rescorer achieves 7\% WER reduction compared to the best LSTM-based deliberation [18] for VS and proper noun recognition, and reduces computation by $38 \%$ relative. Compared to non-deliberation transformer rescoring [24], our deliberation rescorer shows $9 \%$ and $11 \%$ relative improvement. To achieve a tradeoff between WER and computation, we explore reducing the model complexity by encoding a single hypotheses for deliberation, and achieves $4 \%$ and $8 \%$ WER reduction for VS and proper noun recognition, respectively, by using 1.25 times computation of [24].

\section{TRANSFORMER-BASED DELIBERATION}

\subsection{Transformer Deliberation Architecture}

As shown in Fig. 1, our transformer deliberation model has two main parts: A first-pass RNN-T model, and a second-pass transformer deliberation rescorer. The overall deliberation structure resembles that of $[17,18]$. The input of the RNN$\mathrm{T}$ encoder are log-mel filterbank energies, $\mathbf{X}=\left(\mathbf{x}_{1}, \ldots, \mathbf{x}_{T}\right)$, where $T$ denotes the number of frames. The encoder output is then fed to an RNN-T decoder to produce first-pass decoding results $\mathbf{Y}_{r}=\left(\mathbf{y}_{r}^{1}, \ldots, \mathbf{y}_{r}^{B}\right)$ in a streaming fashion, where $B$ is the beam search size. Note our RNN-T decoder is the same as [7] which includes both the prediction and joint networks in [1].

The second-pass model is a multi-source transformer rescorer. The general structure of the transformer rescorer is similar to [22] and consists of $N$ transformer layers, each of which contains self-attention, encoder-decoder attention, and a feed forward layer. Different from [22], our "encoderdecoder" attention attends to two sources. One is the encoder outputs, which are optionally encoded by an additional encoder (dashed box in Fig. 1) to generate audio encoding $\mathbf{E}$, and the other is encoding of first-pass hypotheses. Note that to encode first-pass hypotheses $\mathbf{Y}_{r}$, we still use a bidirectional LSTM since the focus of this work is on the decoder. We encode multiple hypotheses $\left\{\mathbf{y}_{r}^{i}\right\}$ separately using the same bidirectional encoder, where $i=1, \ldots, H$, and $H \leq B$ is the number of hypotheses used. Encoding of multiple hypotheses is then concatenated in time for attention computing. 
Our previous LSTM-based deliberation model [18] did not consider the order of n-best hypotheses during encoding. In this work, we propose to embed the hypothesis order (i.e. hypothesis rank in the n-best list) using a learned embedding [27] and add that to the embedding of each token in the hypothesis. We keep the additional encoder unidirectional due to latency considerations.

The multi-source "encoder-decoder" attention generates two context vectors, and we use a merger layer to combine them. We tried multiple merging options such as concatenation, sum, attention, and gated average [34]. In the attention case, we use the previous self-attention layer output as query and two source context vectors as key and values. For gated average, we take the concatenated source contexts as inputs and uses a softmax layer to predict weights for two sources. The output vector is then a weighted average of the two sources.

For structural comparison, the proposed model uses a transformer-based multi-source decoder for rescoring, instead of a LSTM-based decoder [18]. Compared to [24], our model attends to both encoder outputs and first-pass hypothesis encoding, while [24] only attends to encoder outputs for rescoring.

\subsection{Training and Rescoring}

Similar to [18], we use a two-step training process: Train the RNN-T model first, and then fix the RNN-T parameters and only train the transformer deliberation rescorer and additional encoder layers. In the second pass, for the $u$-th step, the transformer deliberation decoder is trained to pre$\operatorname{dict} p\left(y_{u} \mid \mathbf{X},\left\{\mathbf{y}_{r}^{i}\right\}, y_{1}, \ldots, y_{u-1}\right)$ by using encoder outputs $\mathbf{X}$, first-pass hypotheses $\left\{\mathbf{y}_{r}^{i}\right\}, i=1, \ldots, H$, and all previously predicted tokens using cross-entropy (CE) loss.

Minimum word error rate (MWER) training [33] has been found effective in previous works [7, 18, 24], and thus we fine-tune the rescorer by training using the MWER loss. MWER training optimizes the expected word error rate by using n-best hypotheses:

$$
L_{\mathrm{MWER}}\left(\mathbf{x}, \mathbf{y}^{*}\right)=\sum_{i=1}^{B} \hat{P}\left(\mathbf{y}_{d}^{i} \mid \mathbf{X}, \mathbf{Y}_{r}\right)\left[W\left(\mathbf{y}_{d}^{i}, \mathbf{y}^{*}\right)-\widehat{W}\right]
$$

$\mathbf{y}_{d}^{i}$ is the $i$-th hypothesis from the transformer deliberation decoder. To make the MWER training match rescoring, we compute $P\left(\mathbf{y}_{d}^{i} \mid \mathbf{X}, \mathbf{Y}_{r}\right)$ by rescoring the first-pass hypotheses $\mathbf{Y}_{r}$. In addition, $W\left(\mathbf{y}_{d}^{i}, \mathbf{y}^{*}\right)$ is the number of word errors for $\mathbf{y}_{d}^{i}$ w.r.t the ground truth target $\mathbf{y}^{*}$, and $\widehat{W}$ is the average number of word errors of all n-best hypotheses. $\hat{P}\left(\mathbf{y}_{d}^{i} \mid \mathbf{X}, \mathbf{Y}_{r}\right)$ is the normalized probability of the $i$-th hypothesis over all hypotheses. $B$ is the beam size. In practice, we combine the MWER loss with CE loss to stabilize training:

$$
L_{\mathrm{MWER}}^{\prime}\left(\mathbf{x}, \mathbf{y}^{*}\right)=L_{\mathrm{MWER}}\left(\mathbf{x}, \mathbf{y}^{*}\right)+\alpha L_{\mathrm{CE}}\left(\mathbf{x}, \mathbf{y}^{*}\right)
$$

where $\alpha=0.01$ as in [33].

Similar to [18], we also explore jointly training the model by updating the whole RNN-T model, and the second-pass rescorer in the second step of the training. We show that this further improves the rescoring quality in Sect. 4.5.

Our overall decoding also consists of two passes: 1) The RNN-T model generates the first-pass results $\mathbf{Y}_{r}$, and 2) The transformer deliberation rescorer attends to both $\mathbf{Y}_{r}$ (or a subset) and $\mathbf{E}$ to re-rank first-pass hypotheses to produce $\mathbf{y}_{d}$. In rescoring, we run the deliberation decoder on $\mathbf{Y}_{r}$ in a teacherforcing mode [16, 24]. A major difference from [16, 24] is that deliberation rescoring has access to bi-directional encoding of complete first-pass hypotheses.

\section{EXPERIMENTAL SETUP}

\subsection{Model Details}

We use a domain-id RNN-T [7, 30] as the first-pass model. A domain-id is fed to the RNN-T encoder as a one-hot vector to differentiate 4 domains: Search, farfield, telephony and YouTube. The RNN-T encoder is a 8-layer LSTM [35]. Every LSTM is unidirectional with 2,048 hidden units followed by 640-dimensional projection. A time-reduction layer is added after the second LSTM to increase the inference speed without accuracy loss. The RNN-T decoder contains a prediction network with 2-layer LSTM and a joint-network with a single feed-forward layer of 640 hidden units. The RNN-T decoder is trained to predict 4,096 mixed-case wordpieces [28].

Our transformer deliberation rescorer consists of 4 transformer layers, each of which has a model dimension $d_{\text {model }}=$ 640 and feed forward layer dimension $d_{f f}=2560$, proportional to the sizes in [22]. Both self-attention and "encoderdecoder" attention use multi-headed dot-product attention with 8 heads. We have two sources for the "encoder-decoder" attention and it is applied for all 4 layers. Bidirectional encoding of multiple RNN-T hypotheses are concatenated and used as the second source. The hypotheses are first padded with end-of-sentence label to a length of 120, and then each token in a hypothesis is embedded using the same 640-dimensional embedding layer as in the transformer decoder. Hypothesis order embedding is then added to each token embedding. The embeddings are then encoded by a 2-layer bidirectional LSTM encoder. Each LSTM has 2,048 hidden units and 320dimensional projection. The bidirectional LSTM has a total of $32 \mathrm{M}$ parameters, more than that of the LSTM-based deliberation [18] because we reuse 640-dimensional embedding from the transformer decoder.

The two output context vectors are then summed to produce a single 640-dimensional context vector. Sum is the most effective and efficient among all methods we explored as in Sect. 4.1. In total, the rescorer has $37 \mathrm{M}$ parameters, including a 6.3M-parameter "encoder-decoder" attention on RNN-T hypotheses. The rescorer has a 4,096-dimensional 
softmax layer to predict the same mixed-case wordpieces as the RNN-T.

\subsection{Large Scale Training}

We use the multidomain datasets described in [29] for largescale training. The English utterances are sampled from multiple domains such as general Google traffic, far-field environments, telephony conversations, and YouTube. These utterances are anonymized and hand-transcribed except for YouTube whose transcripts are extracted in a semi-supervised way [36]. We augment the clean training utterances by artificially corrupting them by using a room simulator, varying degrees of noise, and reverberation such that the signal-tonoise ratio (SNR) is between $0 \mathrm{~dB}$ and $30 \mathrm{~dB}$ [37]. We also use mixed-bandwidth utterances at $8 \mathrm{kHz}$ or $16 \mathrm{kHz}$ for training [38].

For the feature extraction front-end, we divide and window a speech waveform using 32-ms hanning windows at a rate of $10 \mathrm{~ms}$, and then compute 128-dimensional log-Mel spectra. Each log-spectrum is stacked with three previous frames to form a 512-dimensional vector, and then downsampled to a $30-\mathrm{ms}$ frame rate. Our models are trained in Tensorflow [39] using the Lingvo framework [40] on $8 \times 8$ TPU slices with a global batch size of 4,096. As in [16], we use constant learning rate for training and maintain an exponential moving average [41] of the trained model parameters for evaluation.

\subsection{Evaluation}

Our main test set is Google Voice Search (VS), which includes $\sim 14 \mathrm{~K}$ anonymized hand-transcribed VS utterances sampled from Google traffic. To measure the model effectiveness on proper noun recognition, we also use a side-by-side $(\mathrm{SxS})$ test set, which contains utterances where the E2E models [16] performs inferior to a state-of-the-art conventional model [13]. A Search domain ID is used for both test sets. In addition to WER, we also report computational complexity in GIGA floating-point operations (GFLOPS) for secondpass decoding. The GFLOPS is computed as total operations needed for bidirectional encoding and second-pass rescoring, and is proportional to beam size $(B)$ and output sequence length $(N)$ :

$$
\mathrm{GFLOPS}=\mathcal{O}(B N)
$$

In our experiments, we use $B=8$ and $N=12$ (wordpiece length for the VS utterance corresponding to 90th percentile rescoring latency [24]).

\section{RESULTS}

We first present ablation studies to find the best architecture for the proposed transformer deliberation rescorer and then compare to the best-performing LSTM-based deliberation rescorer [18] and non-deliberation transformer rescoring [24]. We do not use any voice-activity detector or endpointer [42] in either first-pass decoding or second-pass rescoring.

\subsection{Attention Merger}

We tried 4 different ways, i.e., concatenation, sum, attention, and gated average, to merge the source context vectors in an attention merger layer as shown in Fig. 1. The output is a merged context vector which has the same dimension as the output of the previous self-attention layer. In the concatenation case, we first project each source vector to half of its dimension and then concatenate them. We find that sum and gated average performs the best: 5.7\% WER for VS. We thus choose sum for simplicity and use it for following experiments.

\begin{tabular}{|c|c|c|c|c|}
\hline ID & E1 & E2 & E3 & E4 \\
\hline \hline Attention Merger & Concat & Sum & Atten & Gated \\
\hline VS WER (\%) & 5.8 & 5.7 & 6.1 & 5.7 \\
\hline
\end{tabular}

Table 1. WERs (\%) of transformer deliberation rescoring by using different types of merging layers.

\subsection{Additional Encoder}

Additional encoder (AE) layers (dashed box in Fig. 1) are found to improve recognition for LSTM-based deliberation [18]. The AE consists of a 2-layer LSTM with 2,048 hidden units followed by 640-dimensional projection per layer. In Table 2, we observe that transformer deliberation rescoring also benefits from $\mathrm{AE}$ layers. A rescorer with $\mathrm{AE}$ layers (E5) performs around $4 \%$ relatively better than without (E4).

\begin{tabular}{|c|c|c|}
\hline ID & Model & VS WER (\%) \\
\hline \hline E4 & w/o AE & 5.7 \\
\hline E5 & w/ AE & 5.5 \\
\hline
\end{tabular}

Table 2. WERs (\%) with or without AE layers.

\subsection{Hypothesis Encoding and MWER}

Similar to [18], we also observe improvement by using multiple first-pass hypotheses and MWER training. However, the improvement is smaller compared to our previous study [18], probably because our RNN-T baseline is significantly better (see Table 5). In addition, we find that a 4-hypothesis MWER rescorer already performs the best and thus use it for comparison in Sect. 4.5 considering computational efficiency. The models in Table 3 all have AE layers. 


\begin{tabular}{|c|c|c|c|c|}
\hline ID & E6 & E7 & E8 & E9 \\
\hline \hline Model & 1 hyp & 2 hyp & 4 hyp & 8 hyp \\
\hline Trans. Delib. & 5.5 & 5.5 & 5.4 & 5.4 \\
\hline + MWER & 5.4 & 5.4 & 5.3 & 5.3 \\
\hline
\end{tabular}

Table 3. VS WERs (\%) of transformer deliberation rescoring by attending to different numbers of RNN-T hypotheses and MWER training.

\subsection{Hypothesis Order Embedding}

In this work, we also propose to embed the hypothesis order and add that to the embedding of each wordpiece token in a hypotheses. This let the model differentiate hypotheses in the first-pass n-best list. When using encoding of 4 first-pass hypotheses $(\mathrm{E} 8)$, we find the order embedding slightly improves WER (Table 4).

\begin{tabular}{|c|c|c|}
\hline ID & Model & VS WER (\%) \\
\hline \hline E8 & w/o order embedding & 5.3 \\
\hline E10 & w/ order embedding & 5.2 \\
\hline
\end{tabular}

Table 4. WERs (\%) with or without order embedding.

\subsection{Comparisons}

From the above analysis, we use an MWER trained 4hypothesis transformer deliberation rescorer with additional encoder and order embedding for comparison (E10 in Table 5). In Table 5, we compare deliberation models with a firstpass RNN-T model (B0, [7] without endpointing), LSTM deliberation rescoring (B1) [18], and transformer rescoring (B2, [24] without endpointing).

First, we observe that all two-pass models perform substantially better than their first-pass RNN-T. The relative WER reduction by the proposed transformer deliberation rescorer is around $16 \%$ for VS and $17 \%$ for SxS. Second, comparing transformer (E10) and LAS (B2) deliberation for rescoring, we achieve 5\% relative WER reduction for VS and $4 \%$ for SxS. Joint training (E11) further improves the WER reductions to $7 \%$ relative. We also compute a second-pass GFLOPS as a sum of operations needed by both bidirectional LSTM encoder and deliberation rescorer, and show that the transformer deliberation rescorer reduces GFLOPS by $38 \%$ relative compared to LSTM-based deliberation (from 7.7 to 4.8 GFLOPS in Table 5). Part of the reduction is because we are now using 4 hypotheses in bidirectional encoding, which reduces computation from 3.4 to 1.7 GFLOPS, for bidirectional encoding alone. The other improvement is from the transformer rescorer (4.3 to 3.1 GFLOPS, i.e., $28 \%$ relative reduction).

We also compare our best transformer deliberation rescorer (E11) to a non-deliberation transformer rescorer (B1) in [24], which relies on a single attention on encoder outputs for rescoring. We achieve $9 \%$ and $11 \%$ relative WER reductions for VS and SxS, respectively. As for GFLOPS, our transformer deliberation rescorer has extra bidirectional encoding for first-pass hypotheses and multi-source attention, and is thus 1.7 times of B1. To reduce the computation of hypothesis encoding, we also present a 1-hypothesis transformer deliberation rescorer $(\mathrm{E} 6)$ in Table 5. The model still performs around $4 \%$ and $7 \%$ relatively better than B1, on VS and SxS, respectively, and reduces the computation to 1.25 times. We note that there are potentially more ways to improve computation (and latency) for this model, including using a transformer encoder for hypothesis encoding and parallel rescoring as in [24]. We will leave them as future work.

\section{CONCLUSION}

We presented a transformer deliberation model for rescoring. The best proposed model achieves $18 \%$ and $19 \%$ relative WER reductions for VS and SxS, respectively, compared to the first-pass RNN-T. The reductions are 9\% and $11 \%$ compared to non-deliberation transformer rescoring, and 7\% compared to LSTM-based deliberation. Our transformer deliberation model is more efficient than LSTM deliberation by reducing GFLOPS by $38 \%$ relative, where a $28 \%$ relative reduction is due to the transformer rescorer. We show that a 1hypothesis rescorer further reduces the computation by $27 \%$ while maintaining some WER improvement. In future work, we will explore using transformer encoder for hypothesis encoding and parallel rescoring [24] to improve latency.

\section{ACKNOWLEDGEMENT}

We thank Wei Li, James Qin, and Yanzhang He for their help in computing GFLOPS and useful discussions.

\section{References}

[1] Alex Graves, "Sequence transduction with recurrent neural networks," arXiv preprint arXiv:1211.3711, 2012

[2] Jan K Chorowski, Dzmitry Bahdanau, Dmitriy Serdyuk, Kyunghyun Cho, and Yoshua Bengio, "Attention-based models for speech recognition," in Advances in neural information processing systems, 2015, pp. 577-585.

[3] William Chan, Navdeep Jaitly, Quoc Le, and Oriol Vinyals, "Listen, attend and spell: A neural network for large vocabulary conversational speech recognition," in Proc. ICASSP. IEEE, 2016, pp. 4960-4964.

[4] Jinyu Li, Yu Wu, Yashesh Gaur, Chengyi Wang, Rui Zhao, and Shujie Liu, "On the comparison of popular end-to-end models for large scale speech recognition," arXiv preprint arXiv:2005.14327, 2020.

[5] Yanzhang He, Tara N. Sainath, Rohit Prabhavalkar, Ian McGraw, Raziel Alvarez, Ding Zhao, David Rybach, Anjuli Kannan, Yonghui Wu, Ruoming Pang, et al., "Streaming end-to-end speech recognition for mobile devices," in Proc. ICASSP. IEEE, 2019, pp. 6381-6385. 


\begin{tabular}{|c|c|c|c|c|c|}
\hline \multirow{2}{*}{ ID } & \multirow{2}{*}{ Model } & \# Encoded First-Pass & \multicolumn{2}{|c|}{ WER (\%) } & 2nd Pass \\
\cline { 4 - 6 } & & Hypotheses (H) & VS & SxS & GFLOPS \\
\hline \hline B0 & RNN-T [7] & - & 6.2 & 28.4 & - \\
\hline B1 & Transformer Rescoring [24] & - & 5.6 & 25.9 & 2.8 \\
\hline B2 & LSTM Deliberation Rescoring [18] & 8 & 5.5 & 24.7 & 7.7 \\
\hline E10 & Transformer Deliberation Rescoring & 4 & 5.2 & 23.6 & 4.8 \\
\hline E11 & + Joint Training & 4 & $\mathbf{5 . 1}$ & $\mathbf{2 3 . 0}$ & 4.8 \\
\hline E6 & Transformer Deliberation Rescoring & 1 & 5.4 & 23.9 & 3.5 \\
\hline
\end{tabular}

Table 5. Comparison of RNN-T, non-deliberation transformer rescoring, LSTM-based deliberation, and transformer-based deliberation rescoring models in WERs (\%) and GFLOPS. All two-pass models are augmented with AE layers and trained with MWER loss except the RNN-T model.

[6] Chung-Cheng Chiu, Tara N. Sainath, Yonghui Wu, Rohit Prabhavalkar, Patrick Nguyen, Zhifeng Chen, Anjuli Kannan, Ron J Weiss, Kanishka Rao, Ekaterina Gonina, et al., "State-of-the-art speech recognition with sequence-to-sequence models," in Proc. ICASSP. IEEE, 2018, pp. 4774-4778.

[7] Tara N. Sainath, Yanzhang He, Bo Li, Arun Narayanan, Ruoming Pang, Antoine Bruguier, Shuo-yiin Chang, Wei Li, Raziel Alvarez, Zhifeng Chen, et al., "A streaming on-device end-to-end model surpassing server-side conventional model quality and latency," in 2020 IEEE International Conference on Acoustics, Speech and Signal Processing (ICASSP). IEEE, 2020, pp. 6059-6063.

[8] Dzmitry Bahdanau, Kyunghyun Cho, and Yoshua Bengio, "Neural machine translation by jointly learning to align and translate," arXiv preprint arXiv:1409.0473, 2014.

[9] Ching-Feng Yeh, Jay Mahadeokar, Kaustubh Kalgaonkar, Yongqiang Wang, Duc Le, Mahaveer Jain, Kjell Schubert, Christian Fuegen, and Michael L Seltzer, "Transformer-transducer: End-to-end speech recognition with self-attention," arXiv preprint arXiv:1910.12977, 2019.

[10] Qian Zhang, Han Lu, Hasim Sak, Anshuman Tripathi, Erik McDermott, Stephen Koo, and Shankar Kumar, "Transformer transducer: A streamable speech recognition model with transformer encoders and RNN-T loss," in 2020 IEEE International Conference on Acoustics, Speech and Signal Processing (ICASSP). IEEE, 2020, pp. 7829-7833.

[11] Liang Lu, Changliang Liu, Jinyu Li, and Yifan Gong, "Exploring transformers for large-scale speech recognition," arXiv preprint arXiv:2005.09684, 2020.

[12] Emiru Tsunoo, Yosuke Kashiwagi, and Shinji Watanabe, "Streaming transformer ASR with blockwise synchronous inference," arXiv preprint arXiv:2006.14941, 2020.

[13] Golan Pundak and Tara Sainath, "Lower frame rate neural network acoustic models," in Proc. Interspeech 2016, 2016, pp. 22-26.

[14] Wei Zhou, Wilfried Michel, Kazuki Irie, Markus Kitza, Ralf Schlüter, and Hermann Ney, "The RWTH ASR system for TED-LIUM release 2: Improving hybrid HMM with specaugment," in 2020 IEEE International Conference on Acoustics, Speech and Signal Processing (ICASSP). IEEE, 2020, pp. 7839-7843.

[15] Jinyu Li, Rui Zhao, Zhong Meng, Yanqing Liu, Wenning Wei, Sarangarajan Parthasarathy, Vadim Mazalov, Zhenghao Wang, Lei He, Sheng Zhao, et al., "Developing RNN-T models surpassing highperformance hybrid models with customization capability," arXiv preprint arXiv:2007.15188, 2020.
[16] Tara N. Sainath, Ruoming Pang, David Rybach, Yanzhang He, Rohit Prabhavalkar, Wei Li, Mirkó Visontai, Qiao Liang, Trevor Strohman, Yonghui Wu, Ian McGraw, and Chung-Cheng Chiu, "Two-pass end-toend speech recognition," in Proc. Interspeech 2019, 2019, pp. 27732777.

[17] Yingce Xia, Fei Tian, Lijun Wu, Jianxin Lin, Tao Qin, Nenghai Yu, and Tie-Yan Liu, "Deliberation networks: Sequence generation beyond one-pass decoding," in Advances in Neural Information Processing Systems, 2017, pp. 1784-1794.

[18] Ke Hu, Tara N. Sainath, Ruoming Pang, and Rohit Prabhavalkar, "Deliberation model based two-pass end-to-end speech recognition," in 2020 IEEE International Conference on Acoustics, Speech and Signal Processing (ICASSP). IEEE, 2020, pp. 7799-7803.

[19] R. Ma, H. Li, Q. Liu, L. Chen, and K. Yu, "Neural lattice search for speech recognition," in 2020 IEEE International Conference on Acoustics, Speech and Signal Processing (ICASSP), 2020, pp. 7794-7798.

[20] Cal Peyser, Hao Zhang, Tara N. Sainath, and Zelin Wu, "Improving performance of end-to-end ASR on numeric sequences," in Proc. Interspeech 2019, 2019, pp. 2185-2189.

[21] Jinxi Guo, Tara N. Sainath, and Ron J Weiss, "A spelling correction model for end-to-end speech recognition," in Proc. ICASSP. IEEE, 2019, pp. 5651-5655.

[22] Ashish Vaswani, Noam Shazeer, Niki Parmar, Jakob Uszkoreit, Llion Jones, Aidan N Gomez, Lukasz Kaiser, and Illia Polosukhin, "Attention is all you need," in Advances in neural information processing systems, 2017, pp. 5998-6008.

[23] Yongqiang Wang, Abdelrahman Mohamed, Duc Le, Chunxi Liu, Alex Xiao, Jay Mahadeokar, Hongzhao Huang, Andros Tjandra, Xiaohui Zhang, Frank Zhang, et al., "Transformer-based acoustic modeling for hybrid speech recognition," arXiv preprint arXiv:1910.09799, 2019.

[24] Wei Li, James Qian, Chung-Cheng Chiu, Ruoming Pang, and Yanzhang $\mathrm{He}$, "Parallel rescoring with transformer for streaming on-device speech recognition," in Proc. Interspeech. IEEE, 2020 (submitted).

[25] Oleksii Hrinchuk, Mariya Popova, and Boris Ginsburg, "Correction of automatic speech recognition with transformer sequence-to-sequence model," in 2020 IEEE International Conference on Acoustics, Speech and Signal Processing (ICASSP). IEEE, 2020, pp. 7074-7078.

[26] Shigeki Karita, Nanxin Chen, Tomoki Hayashi, Takaaki Hori, Hirofumi Inaguma, Ziyan Jiang, Masao Someki, Nelson Enrique Yalta Soplin, Ryuichi Yamamoto, Xiaofei Wang, et al., "A comparative study on transformer vs RNN in speech applications," arXiv preprint arXiv:1909.06317, 2019. 
[27] Ehsan Variani, Tongzhou Chen, James Apfel, Bhuvana Ramabhadran, Seungji Lee, and Pedro Moreno, "Neural oracle search on n-best hypotheses," in 2020 IEEE International Conference on Acoustics, Speech and Signal Processing (ICASSP). IEEE, 2020, pp. 7824-7828.

[28] Mike Schuster and Kaisuke Nakajima, "Japanese and Korean voice search,” in Proc. ICASSP. IEEE, 2012, pp. 5149-5152.

[29] Arun Narayanan, Rohit Prabhavalkar, Chung-Cheng Chiu, David Rybach, Tara N. Sainath, and Trevor Strohman, "Recognizing longform speech using streaming end-to-end models," in 2019 IEEE Automatic Speech Recognition and Understanding Workshop (ASRU). IEEE, 2019, pp. 920-927.

[30] Bo Li, Tara N Sainath, Khe Chai Sim, Michiel Bacchiani, Eugene Weinstein, Patrick Nguyen, Zhifeng Chen, Yanghui Wu, and Kanishka Rao, "Multi-dialect speech recognition with a single sequence-tosequence model," in 2018 IEEE international conference on acoustics, speech and signal processing (ICASSP). IEEE, 2018, pp. 4749-4753.

[31] John J Godfrey, Edward C Holliman, and Jane McDaniel, "Switchboard: Telephone speech corpus for research and development," in Acoustics, Speech, and Signal Processing, IEEE International Conference on. IEEE Computer Society, 1992, vol. 1, pp. 517-520.

[32] Christopher Cieri, David Miller, and Kevin Walker, "The Fisher corpus: a resource for the next generations of speech-to-text.," in LREC, 2004, vol. 4, pp. 69-71.

[33] Rohit Prabhavalkar, Tara N. Sainath, Yonghui Wu, Patrick Nguyen, Zhifeng Chen, Chung-Cheng Chiu, and Anjuli Kannan, "Minimum word error rate training for attention-based sequence-to-sequence models," in Proc. ICASSP. IEEE, 2018, pp. 4839-4843.

[34] Ankur Bapna and Orhan Firat, "Non-parametric adaptation for neural machine translation," in Proc. 2019 Conference of the North American Chapter of the Association for Computational Linguistics (NAACL), Minneapolis, Minnesota, USA, 2019, pp. 1921-1931.

[35] Sepp Hochreiter and Jürgen Schmidhuber, "Long short-term memory," Neural computation, vol. 9, no. 8, pp. 1735-1780, 1997.

[36] Hank Liao, Erik McDermott, and Andrew Senior, "Large scale deep neural network acoustic modeling with semi-supervised training data for YouTube video transcription," in 2013 IEEE Workshop on Automatic Speech Recognition and Understanding. IEEE, 2013, pp. 368373.

[37] Chanwoo Kim, Ananya Misra, Kean Chin, Thad Hughes, Arun Narayanan, Tara N. Sainath, and Michiel Bacchiani, "Generation of large-scale simulated utterances in virtual rooms to train deep-neural networks for far-field speech recognition in Google home," in Proc. Interspeech, 2017, pp. 379-383.

[38] Dong Yu, Michael L Seltzer, Jinyu Li, Jui-Ting Huang, and Frank Seide, "Feature learning in deep neural networks-studies on speech recognition tasks," arXiv preprint arXiv:1301.3605, 2013.

[39] Martín Abadi, Paul Barham, Jianmin Chen, Zhifeng Chen, Andy Davis, Jeffrey Dean, Matthieu Devin, Sanjay Ghemawat, Geoffrey Irving, Michael Isard, et al., "TensorFlow: A system for large-scale machine learning," in 12th USENIX Symposium on Operating Systems Design and Implementation (OSDI 16), 2016, pp. 265-283.

[40] Jonathan Shen, Patrick Nguyen, Yonghui Wu, Zhifeng Chen, Mia X Chen, Ye Jia, Anjuli Kannan, Tara Sainath, Yuan Cao, Chung-Cheng Chiu, et al., "Lingvo: A modular and scalable framework for sequenceto-sequence modeling," arXiv preprint arXiv:1902.08295, 2019.

[41] Boris T Polyak and Anatoli B Juditsky, "Acceleration of stochastic approximation by averaging," SIAM journal on control and optimization, vol. 30, no. 4, pp. 838-855, 1992.
[42] Shuo-Yiin Chang, Rohit Prabhavalkar, Yanzhang He, Tara N Sainath, and Gabor Simko, "Joint endpointing and decoding with end-to-end models," in ICASSP 2019-2019 IEEE International Conference on Acoustics, Speech and Signal Processing (ICASSP). IEEE, 2019, pp. 5626-5630. 